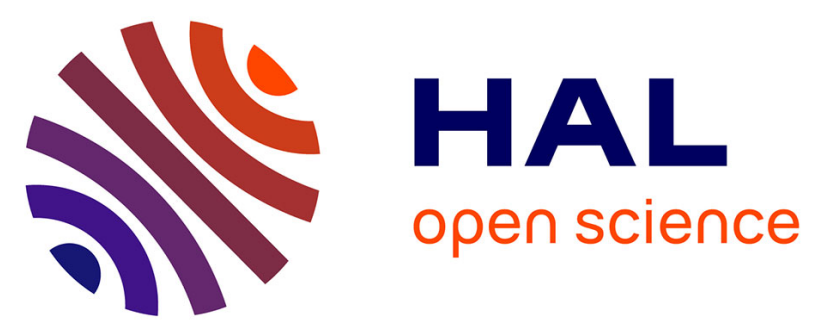

\title{
Investigation of the phase velocities of guided acoustic waves in soft porous layers
}

Laurens Boeckx, Philippe Leclaire, Poonam Khurana, Christ Glorieux, Walter Lauriks, Jean-François Allard

\section{- To cite this version:}

Laurens Boeckx, Philippe Leclaire, Poonam Khurana, Christ Glorieux, Walter Lauriks, et al.. Investigation of the phase velocities of guided acoustic waves in soft porous layers. Journal of the Acoustical Society of America, 2005, pp.545-554. 10.1121/1.1847848 . hal-01326767

\section{HAL Id: hal-01326767 \\ https://hal.science/hal-01326767}

Submitted on 5 Jun 2016

HAL is a multi-disciplinary open access archive for the deposit and dissemination of scientific research documents, whether they are published or not. The documents may come from teaching and research institutions in France or abroad, or from public or private research centers.
L'archive ouverte pluridisciplinaire HAL, est destinée au dépôt et à la diffusion de documents scientifiques de niveau recherche, publiés ou non, émanant des établissements d'enseignement et de recherche français ou étrangers, des laboratoires publics ou privés. 


\title{
Investigation of the phase velocities of guided acoustic waves in soft porous layers
}

\author{
L. Boeckx, P. Leclaire, P. Khurana, C. Glorieux, and W. Lauriks \\ Laboratorium voor Akoestiek en Thermische Fysica, Katholieke Universiteit Leuven, Celestijnenlaan 200D, \\ B-3001 Heverlee, Belgium
}

\author{
J. F. Allard \\ Laboratoire d'Acoustique de l'Université du Maine, UMR CNRS 6613, Avenue Olivier Messiaen, \\ 72085 Le Mans Cédex, France
}

\begin{abstract}
A new experimental method for measuring the phase velocities of guided acoustic waves in soft poroelastic or poroviscoelastic plates is proposed. The method is based on the generation of standing waves in the material and on the spatial Fourier transform of the displacement profile of the upper surface. The plate is glued on a rigid substrate so that it has a free upper surface and a nonmoving lower surface. The displacement is measured with a laser Doppler vibrometer along a line corresponding to the direction of propagation of plane surface waves. A continuous sine with varying frequencies was chosen as excitation signal to maximize the precision of the measurements. The spatial Fourier transform provides the wave numbers, and the phase velocities are obtained from the relationship between wave number and frequency. The phase velocities of several guided modes could be measured in a highly porous foam saturated by air. The modes were also studied theoretically and, from the theoretical results, the experimental results, and a fitting procedure, it was possible to determine the frequency behavior of the complex shear modulus and of the complex Poisson ratio from $200 \mathrm{~Hz}$ to $1.4 \mathrm{kHz}$, in a frequency range higher than the traditional methods.
\end{abstract}

\section{INTRODUCTION}

The acoustical properties of porous materials can generally be well described in a wide frequency range with Biot's theory, ${ }^{1}$ which involves the determination of a number of physical parameters. In many cases for air-saturated materials, the porous frame can be considered to be much more rigid and heavier than air, and a simplified model can be used in the rigid frame approximation. Many studies have been successfully carried out in this approximation and are reported in Ref. 2. There is also a growing interest in applications where the frequency dependence of the mechanical rigidity of the material should be taken into account in the full Biot theory for poroelastic and poroviscoelastic media., ${ }^{3,4,2}$ However, the main limitation of the full model is the lack of data on the dynamic rigidities of the porous frame. Classical methods for determining these parameters involve the application of vibrations to samples ${ }^{5-10}$ (e.g., rods, cubes, or small plates) of finite sizes with respect to the wavelengths involved. The properties (resonance frequencies, damping, dynamic behavior) of the transfer function between the response and the excitation yield the mechanical properties of the excited structure. However, the frequency range described in these methods is limited to the lower part of the audible frequency range (typically below $400 \mathrm{~Hz}$ ). A new method for measuring the shear modulus of air-filled porous materials based on the propagation of Rayleigh waves in thick layers was recently proposed by Allard et al. ${ }^{11}$ and provided useful information above $3 \mathrm{kHz}$. More recently, Allard et al. ${ }^{12}$ have proposed a new method of measurement in thinner samples in which Biot's shear wave is excited. This method is based on the effect of the resonance of the porous frame around the quarter shear wavelength on the pole of the reflection coefficient.

Surface waves at the interface between a fluid and a porous solid have been studied theoretically by many authors and in particular by Deresiewicz, ${ }^{13}$ and Feng and Johnson ${ }^{14}$ for liquid saturated media. The surface wave in the air above a porous absorbing material and rough surfaces was studied theoretically and experimentally by Attenborough, ${ }^{15,16}$ Attenborough and Chen, ${ }^{17}$ Lauriks et al. ${ }^{18}$ Kelders et al.,${ }^{19}$ and Allard et al. ${ }^{20}$

In this article, we investigate the propagation of guided waves in a layer of porous material in a wide frequency range, from the typical frequencies of the classical vibrational methods to the high-frequency limit of the Rayleigh wave. First results of this work were presented in Kyoto. ${ }^{21}$ Together with a complete model for guided waves in a soft porous material on a rigid substrate, a new experimental method is proposed in this article for the determination of the modes of propagation in a plate of finite sizes. This method, characterized by an increased signal-to-noise ratio and measurement accuracy, is based on the generation of standing waves in the layer of porous material. Lamb waves in plates with free surfaces, and other surface waves in layers or at interfaces have been extensively studied and led to many 


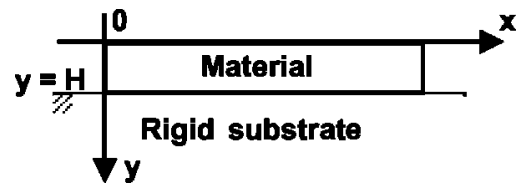

FIG. 1. Layer of material on a rigid substrate and system of coordinates.

applications from material characterization at ultrasonic frequencies ${ }^{22}$ to the oil industry. Theoretical references in this area are provided by the books by Royer and Dieulesaint, ${ }^{23}$ Viktorov, ${ }^{24}$ and Ewing, Jardetsky, and Press. ${ }^{25}$ The theoretical results of our study are presented in the next section. The experimental principle of the new method for measuring the phase velocities of the different modes in of porous layers is presented in Sec. III and applied in Sec. IV to a melamine foam. The phase velocities of two modes were measured and used as reference velocities for the fitting of the dispersion curves leading to the determination of the frequency variations of the complex shear modulus and Poisson ratio.

\section{GUIDED WAVES IN A LAYER OF POROUS MATERIAL}

Results for a nonporous solid of thickness $H$ lying on a rigid substrate are presented first. This simple case will be very useful to the study of the porous case in providing the general shape of the curves, the cutoff frequencies, and the limit velocities that should be expected. Many porous materials in practical applications have a high porosity, a low flow resistivity, and a tortuosity close to 1 . For these materials the couplings are relatively low, and the model for nonporous material will reasonably approximate the porous case. The full model for porous layers on a rigid substrate is presented in Sec. II B, which better describes materials for which the couplings are higher.

\section{A. Guided waves in a layer of soft elastic material on a rigid substrate}

Ewing, Jardetsky, and Press ${ }^{25}$ (p. 189) have studied surface waves in a solid layer over a semi-infinite solid. The frequency spectrum at ultrasonic frequencies of the surface acoustic wave in a solid film over a solid substrate was also studied analytically by Gusev and Hess. ${ }^{26}$ The configuration for a soft, solid layer lying on a rigid substrate is shown in Fig. 1. Following the standard derivation, by choosing the potentials to be linear combinations of sines and cosines and from the application of the appropriate boundary conditions on stresses and displacements at the interfaces, the following dispersion equation is obtained:

$$
\begin{gathered}
-4 k^{2}\left(k^{2}-q^{2}\right)-\sin p H \sin q H\left[\frac{k^{2}}{p q}\left(k^{2}-q^{2}\right)^{2}+4 k^{2} p q\right] \\
+\cos p H \cos q H\left[4 k^{4}+\left(k^{2}-q^{2}\right)^{2}\right]=0,
\end{gathered}
$$

where $k, p$, and $q$ are the wave numbers defined in the classical Lamb theory. Figure 2(a) shows an example of dispersion curves obtained from a numerical search of the roots of Eq. (1). The usual Lamb dispersion curves are shown in Fig. 2(b) for the same material but for a plate of thickness
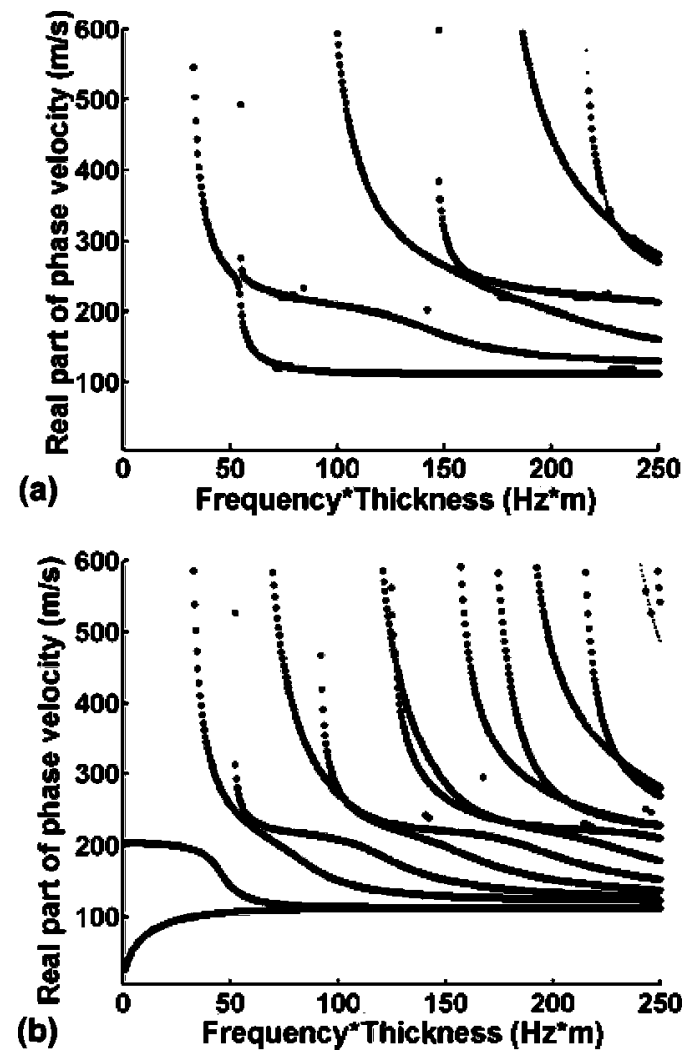

FIG. 2. Phase velocities vs frequency $\times$ thickness for (a) a plate of thickness $H$ of an elastic material on a rigid substrate; (b) a Lamb plate of thickness $2 H$. In both cases the material density is $14 \mathrm{~kg} / \mathrm{m}^{3}, V_{L}=222 \mathrm{~m} / \mathrm{s}$, and $V_{T}$ $=122 \mathrm{~m} / \mathrm{s}$.

$2 H$. The main difference with the Lamb plate is the absence of modes without cutoff frequency and a greater spacing between the modes associated with the change in thickness. Since the thickness always appears in a product with the wave number or with the frequency in the dispersion equation, doubling (halving) the thickness is equivalent to compressing (expanding) the frequency scale. The cutoff frequencies are given by

$$
\begin{aligned}
& \omega_{c}=(2 m+1) \frac{\pi V_{T}}{2 H} \text { and } \omega_{c}=(2 m+1) \frac{\pi V_{L}}{2 H}, \\
& m=0,1,2, \ldots,
\end{aligned}
$$

where $V_{L}$ and $V_{T}$ are, respectively, the velocities of the bulk compressional and shear waves in the material. The study of the high-frequency behavior shows that the first mode tends to the Rayleigh mode and that the velocities of higher order modes tend to the bulk shear velocity.

The results presented above do not account for the presence of a fluid surrounding the layer. The effect of the fluid can be included by replacing the zero in the right-hand side of the equation of dispersion by a fluid term (see Viktorov, ${ }^{24}$ p. 117 for the Lamb case). The main influence of the presence of a loading fluid is the possible existence of a fluid wave that is damped along the $y$ axis, hence localized above the surface (Scholte wave) and/or the possibility for the modes in the layer to "leak out" (radiate) energy in the fluid (leaky Rayleigh wave or leaky Lamb modes). A large body of literature is dedicated to the study of ultrasonic surface 
waves in fluid-loaded media. The reader is referred to the thesis by Van de Rostyne ${ }^{27}$ for an extensive and recent review. The fluid is air in our study and its influence has been neglected in the case of guided waves in nonporous layers on a rigid substrate. However, air is accounted for in the following section where a porous material is studied.

\section{B. Guided waves in a porous layer on a rigid substrate and saturated by air}

The configuration examined in the previous section involves boundary conditions that are of great interest for the study of highly porous sound-absorbing materials. These very soft materials have a Young's modulus between $10^{4}$ and to $10^{6} \mathrm{~Pa}$, typically. For these materials the rigid substrate or the Lamb boundary conditions are fairly easy to set up experimentally and to control.

\section{Biot's equations of poroelasticity}

In Biot's linear equations of poroelasticity ${ }^{28}$ (Biot's second formulation is used here), the total stress tensor $\tau_{i j}$ and the fluid pressure $P_{f}$ in the pores are given by

$$
\begin{aligned}
\tau_{i j} & =2 \mu \varepsilon_{i j}+\delta_{i j}\left(\lambda_{c} \varepsilon-\alpha M \zeta\right), \\
P_{f} & =-\alpha M \varepsilon+M \zeta,
\end{aligned}
$$

with

$$
\begin{aligned}
& \lambda_{c}=\lambda+\alpha^{2} M, \\
& \varepsilon_{i j}=\frac{1}{2}\left(u_{i, j}+u_{j, i}\right), \\
& \varepsilon=\varepsilon_{11}+\varepsilon_{22}+\varepsilon_{33}=\operatorname{div} \boldsymbol{u}, \\
& \zeta=-\operatorname{div} w, \\
& \boldsymbol{w}=\phi(\boldsymbol{U}-\boldsymbol{u}) .
\end{aligned}
$$

In these equations, $\lambda$ and $\mu$ are the Lamé constants of the porous frame, $\alpha$ and $M$ are, respectively, an elastic coupling factor and a rigidity associated with the fluid. They were both defined by Biot and Willis. ${ }^{29} \varepsilon_{i j}$ is the strain tensor of the solid and depends on the solid displacement $\boldsymbol{u} . \varepsilon$ is the solid dilatation, $\phi$ the porosity, $\zeta$ the fluid content, and $w$ corresponds to the displacement of the fluid $\boldsymbol{U}$ relative to the solid.

\section{Boundary conditions}

The porous plate is set in the same configuration as the elastic plate of Sec. II A and the system of coordinates is unchanged. In this configuration, the boundary conditions are expressed as follows:

At $y=0$ :

$$
\tau_{12}=0
$$

$\tau_{22}=-p$ where $p$ is the pressure of the fluid surrounding the layer,

$$
P_{f}=p,
$$

$u_{y}+w_{y}=U_{f}$ where $U_{f}$ is the displacement of matter in the surrounding fluid.

$$
\text { At } \begin{aligned}
y=H: & \\
u_{y} & =0, \\
u_{x} & =0, \\
w_{x} & =0 .
\end{aligned}
$$

\section{Choice of a functional form for the displacement} potentials

Two compressional waves and one shear wave can propagate in fluid-saturated porous material, and the displacements of matter are written in terms of two scalar potentials $\varphi_{1}$ and $\varphi_{2}$ and one vector potential $\psi$

$$
\begin{aligned}
\boldsymbol{u}= & \nabla \varphi_{1}+\nabla \varphi_{2}+\nabla \times \psi, \\
\boldsymbol{w}= & \phi(\boldsymbol{U}-\boldsymbol{u})=\phi \nabla \varphi_{1}\left(\mu_{1}-1\right)+\phi \nabla \varphi_{2}\left(\mu_{2}-1\right) \\
& +\phi \nabla \times \psi\left(\mu_{3}-1\right),
\end{aligned}
$$

where the coefficients $\mu_{1}, \mu_{2}$, and $\mu_{3}$ correspond to the amplitude ratios of the waves in the porous material ${ }^{3,11}$

$$
\begin{aligned}
& \mu_{i}=\frac{P k_{i}-\omega^{2} \rho_{11}}{\omega^{2} \rho_{12}-Q k_{i}}, i=1,2 \\
& \mu_{3}=\frac{\rho_{12}}{\rho_{22}} .
\end{aligned}
$$

In these expressions $k_{i}(i=1,2)$ are the wave numbers of the Biot compressional waves. The elastic coefficients $P$ and $Q$ were defined by Biot and Willis. ${ }^{29}$ These can be related to $\lambda$, $\mu$, to the rigidity of the fluid $K_{f}$ and of the solid $K_{s}$ and to the porosity $\phi$. The following approximation can be used for highly porous materials saturated by air: ${ }^{3}$

$$
\begin{aligned}
& P \approx \frac{2}{3} \mu+\lambda+\frac{(1-\phi)}{\phi} K_{f}, \\
& Q \approx K_{f}(1-\phi) .
\end{aligned}
$$

The following relations are also needed: ${ }^{29}$

$$
\begin{aligned}
& \alpha=\left(1-\frac{Q}{R}\right) \phi \\
& \text { and } M=\frac{R}{Q^{2}},
\end{aligned}
$$

with $R \approx \phi K_{f}$ in the approximation of an air saturated highly porous material. ${ }^{3} \rho_{11}, \rho_{12}, \rho_{22}$ are the coefficients of a density matrix and are functions of the tortuosity $\alpha_{\infty}$

$$
\begin{aligned}
& \rho_{11}=(1-\phi) \rho_{s}+\left(\alpha_{\infty}-1\right) \phi \rho_{f}, \\
& \rho_{12}=-\left(\alpha_{\infty}-1\right) \phi \rho_{f}, \\
& \rho_{22}=\alpha_{\infty} \phi \rho_{f} .
\end{aligned}
$$

The velocities of the two compressional and of the shear waves were given by Biot. ${ }^{1,28}$ They are obtained from 


$$
\begin{aligned}
& \frac{1}{V_{L i}^{2}}=\frac{1}{2} \frac{P \rho_{22}+R \rho_{11}-2 Q \rho_{12}}{P R-Q^{2}}\left[1 \pm\left(1-4 \frac{\left(P R-Q^{2}\right)\left(\rho_{11} \rho_{22}-\rho_{12}^{2}\right)}{\left(P \rho_{22}+R \rho_{11}-2 Q \rho_{12}\right)^{2}}\right)^{1 / 2}\right], \quad i=1,2 \\
& V_{T}=\sqrt{\frac{\mu}{(1-\phi) \rho_{s}+\left(1-\frac{1}{\alpha_{\infty}}\right) \phi \rho_{f}}}
\end{aligned}
$$

The wave attenuation by viscous frictions and thermal exchanges between the solid and the fluid can be formulated through the use of a complex dynamic tortuosity $\alpha(\omega)$ to replace $\alpha_{\infty}$ and of a complex air compressibility (see Ref. 2). These involve the flow resistivity $\sigma$ of the porous material and the viscous and thermal lengths $\Lambda$ and $\Lambda^{\prime}$. For the first three potentials, a linear combination of sines and cosines is chosen

$$
\begin{aligned}
& \varphi_{1}=\left(A_{1} \cos p_{1} y+A_{2} \sin p_{1} y\right) e^{i(\omega t-k x)}, \\
& \varphi_{2}=\left(B_{1} \cos p_{2} y+B_{2} \sin p_{2} y\right) e^{i(\omega t-k x)}, \\
& \psi=\left(C_{1} \cos q y+C_{2} \sin q y\right) e^{i(\omega t-k x)}, \\
& \varphi_{f}=D e^{\gamma y} e^{i(\omega t-k x)} \text { for } y \leqslant 0,
\end{aligned}
$$

where $A_{1}, A_{2}, B_{1}, B_{2}, C_{1}, C_{2}$, and $D$ are constants. $\omega$ is the angular frequency and $t$ is the time. The potentials depend on $x$ and $y$ and satisfy the following equations of propagation:

$$
\begin{aligned}
& \nabla^{2} \varphi_{1}-\frac{1}{V_{L 1}^{2}} \frac{\partial^{2} \varphi_{1}}{\partial t^{2}}=0, \\
& \nabla^{2} \varphi_{2}-\frac{1}{V_{L 2}^{2}} \frac{\partial^{2} \varphi_{2}}{\partial t^{2}}=0 \\
& \text { and } \nabla^{2} \psi-\frac{1}{V_{T}^{2}} \frac{\partial^{2} \psi}{\partial t^{2}}=0 .
\end{aligned}
$$

Harmonic solutions in $\omega$ and $k$ yield for a propagation along $x$

$$
\begin{aligned}
& \frac{\partial^{2} \varphi_{1}}{\partial y^{2}}+p_{1}^{2} \varphi_{1}=0, \\
& \frac{\partial^{2} \varphi_{2}}{\partial y^{2}}+p_{2}^{2} \varphi_{2}=0, \\
& \text { and } \frac{\partial^{2} \psi}{\partial y^{2}}+q^{2} \psi=0,
\end{aligned}
$$

where the coefficients $p_{1}, p_{2}$, and $q$ are wave numbers associated with the propagation of the fast, slow, and shear waves, respectively

$$
\begin{aligned}
& p_{1}^{2}=\frac{\omega^{2}}{V_{L 1}^{2}}-k^{2}, \\
& p_{2}^{2}=\frac{\omega^{2}}{V_{L 2}^{2}}-k^{2},
\end{aligned}
$$

$$
\text { and } q^{2}=\frac{\omega^{2}}{V_{T}^{2}}-k^{2}
$$

The fluid surrounding the layer is included in the model, and a potential $\varphi_{f}$, associated with the wave in the fluid must also be defined. This potential satisfies

$$
\begin{aligned}
& \nabla^{2} \varphi_{f}-\frac{1}{V_{f}^{2}} \frac{\partial^{2} \varphi_{f}}{\partial t^{2}}, \\
& \frac{\partial^{2} \varphi_{f}}{\partial y^{2}}-\gamma^{2} \varphi_{f}=0 .
\end{aligned}
$$

The dependence on $y$ of $\varphi_{f}$ is such that the $y$ component of the propagation constant $\gamma$ is given by

$$
\gamma^{2}=k^{2}-\frac{\omega^{2}}{V_{f}^{2}},
$$

where $V_{f}$ is the free velocity in the fluid. Referring to discussion of Sec. II A and to the form chosen for the potential in Eq. (22), the main features of the components of the wave numbers of the wave in the surrounding fluid are the following:

(i) $\operatorname{Im}(k) \geqslant 0$. This condition should always be fulfilled and will insure that the amplitude of the wave decreases as $x$ increases (or is constant in the undamped case). The definition used for $k$ is $k=\operatorname{Re}(k)+i \operatorname{Im}(k)$.

(ii) $\operatorname{Re}(\gamma)>0$. This corresponds to a fluid wave localized above the interface, i.e., a Scholte wave (semi-infinite solid) or an $A$ wave (plate). If $\operatorname{Im}(k)=0$ in condition (i) the modes are undamped along $x$.

(iii) $\operatorname{Re}(\gamma)=0$ and $\operatorname{Im}(\gamma)>0$. This situation is one where the wave can propagate in the entire fluid half-space and is not localized near the interface. This is made possible if both the radiation condition $\operatorname{Re}(\gamma)=0$ and the causality requirement $\operatorname{Im}(\gamma)>0$ for a wave to travel from the interface and not toward it are fulfilled. In this case the guided modes in the layer will radiate or leak out energy in the fluid. Since $\gamma$ $=\omega \sqrt{1 / V^{2}-1 / V_{f}^{2}}$, the consequences of these conditions on the velocities are that the phase velocity of the guided wave must be greater than the free velocity of the fluid for the condition to be fulfilled. The wave will be radiative for supersonic guided modes. The nature of the fluid mode (localized or radiative) can also change with frequency as the phase velocity varies and can cross the value $V_{f}$. 


\section{Dispersion equation}

The displacements $u_{x}$ and $u_{y}$ are obtained from writing the spatial derivatives of the potentials in Eq. (9)

$$
\begin{aligned}
& u_{x}=\frac{\partial \varphi_{1}}{\partial x}+\frac{\partial \varphi_{2}}{\partial x}+\frac{\partial \psi}{\partial y}, \\
& \text { and } u_{y}=\frac{\partial \varphi_{1}}{\partial y}+\frac{\partial \varphi_{2}}{\partial y}-\frac{\partial \psi}{\partial y} .
\end{aligned}
$$

Similarly, the components of the relative displacement $w$ are obtained from Eq. (10). The other parameters and the useful elements of the stress and strain tensors are calculated from Eqs. (3) to (8). After expressing the boundary conditions given in Sec. II B 2, a $7 \times 7$ determinant is found

$$
\left|\begin{array}{ccccccc}
0 & D_{12} & 0 & D_{14} & D_{15} & 0 & 0 \\
D_{21} & 0 & D_{23} & 0 & 0 & D_{26} & D_{27} \\
D_{31} & 0 & D_{33} & 0 & 0 & 0 & D_{37} \\
0 & D_{42} & 0 & D_{44} & D_{45} & 0 & D_{47} \\
D_{51} & D_{52} & D_{53} & D_{54} & D_{55} & D_{56} & 0 \\
D_{61} & D_{62} & D_{63} & D_{64} & D_{65} & D_{66} & 0 \\
D_{71} & D_{72} & D_{73} & D_{74} & D_{75} & D_{76} & 0
\end{array}\right|=0 .
$$

The coefficients of the matrix are given in the Appendix. Finding the zeros of this determinant, i.e., solving the equation

$$
\operatorname{Det}(\omega, k, H, \ldots)=0,
$$

provides a relationship between $\omega$ and $k$, the equation of dispersion. This relationship is not explicit and must be determined numerically. In general, this determinant has complex values and Eq. (30) should also be solved in the complex plane to account for attenuation mechanisms. This means that the initial values of $k$ must be complex in the numerical algorithm to solve Eq. (30). The theoretical calculation of the dispersion curves for a highly porous melamine

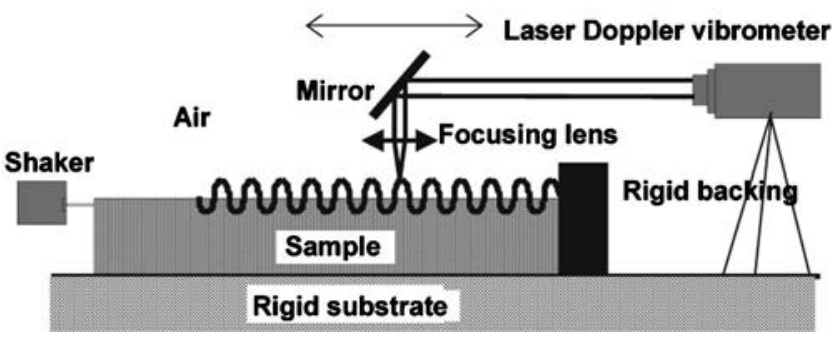

FIG. 3. Experimental setup for the generation and detection of standing waves in a layer of material on a rigid substrate.

foam and an application to the evaluation of the dynamic complex elastic moduli are proposed in Sec. IV.

\section{EXPERIMENTAL PRINCIPLE FOR MEASURING THE PHASE VELOCITIES OF GUIDED ACOUSTIC MODES IN POROUS MEDIA}

\section{A. Experimental setup}

The experimental principle is described in Fig. 3, where a layer of porous material is glued on a plane rigid substrate so that the displacement of the porous frame is zero at the interface between the material and the substrate. Doublefaced tape and a mounting spray were used, in order to obtain uniform boundary conditions over the whole interface. An optical table with a rigidity and a density much greater than that of the porous layer was used as a semi-infinite rigid substrate. The porous layer was excited at one end with a thin aluminum strip used as a line source attached to a shaker at one end and to the sample at the other end with the help of double-faced tape. The shaker was fed with a continuous sinusoidal signal provided by the function generator unit of an SRS SR780 2-channel signal analyzer, and the frequency could be varied. The signal analyzer incorporates a lock-in amplifier. The other end of the layer was glued on a rigid end so that incident waves could be reflected. The difference in impedance is large and it can be assumed that the reflection coefficient at the interface between the rigid end and the (a)

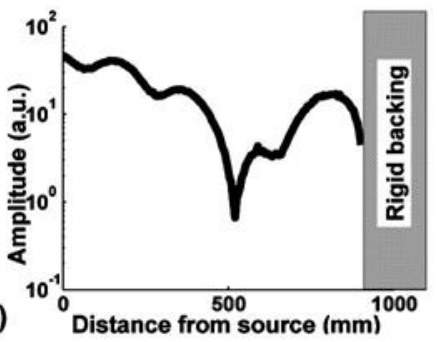

(d)

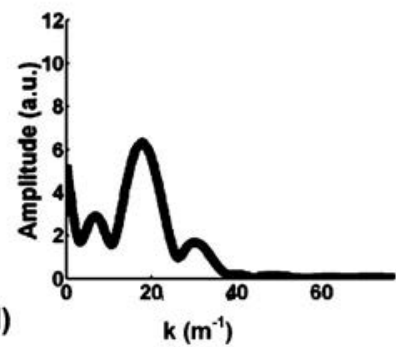

(b)

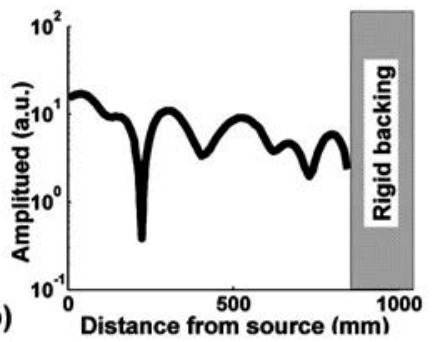

(e)

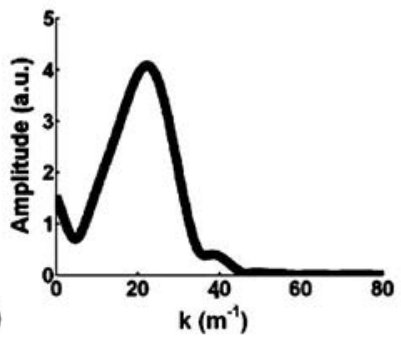

(c)

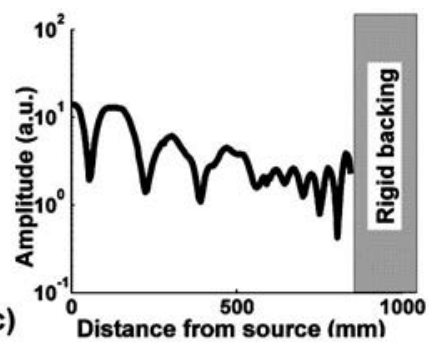

(f)

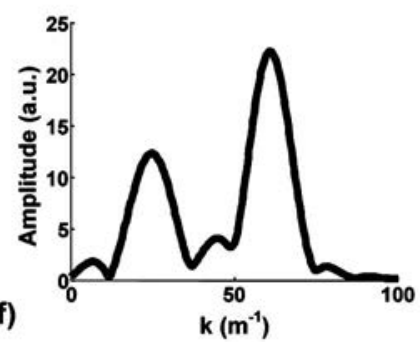

FIG. 4. (a), (b), and (c): Experimental standing wave patterns. (d), (e), and (f): Spatial Fourier transforms of the measured standing wave patterns. (a) and (d) $256 \mathrm{~Hz}$; (b) and (e) $424 \mathrm{~Hz}$; (c) and (f) $1041 \mathrm{~Hz}$. 
TABLE I. Phase velocities of the surface modes in a layer of material on a rigid substrate at different frequencies.

\begin{tabular}{cccccc}
\hline \hline & \multicolumn{5}{c}{ Phase velocities of modes $(\mathrm{m} / \mathrm{s})$} \\
\cline { 2 - 5 } Freq $(\mathrm{Hz})$ & & & & \\
\hline 1041 & 933 & 266 & 146 & 108 & 84 \\
424 & 120 & 69 & & & \\
256 & 253 & 91 & 50 & \\
\hline \hline
\end{tabular}

plate is 1 . The displacement of the surface of the layer was measured with the help of a laser vibrometer. The measurement point on the surface was allowed to move along a line parallel to the $x$ axis. The laser beam at the output of the laser was collimated and a mirror/lens arrangement insured that the beam was always focused on the surface of the material at any position of the beam. Reflection of the laser beam on the material was achieved with the help of a strip of reflective tape in the path of the scanning beam. The entire setup was automated and placed in a semianechoic chamber. For each frequency, the path was scanned with a typical step of 1 to $5 \mathrm{~mm}$ and the amplitude and phase of the signal were recorded at each position. Once the data were recorded, the spatial Fourier transform of the displacement profile in the vicinity of the rigid end was calculated.

\section{B. Standing wave pattern, spatial Fourier transform and phase velocities}

Figure 4 shows the standing wave patterns measured at 256, 424, and $1041 \mathrm{~Hz}$, and the amplitude of their respective Fourier transforms plotted in arbitrary units. The experimental data processing scheme is the following: the spatial Fourier transform $A\left(k, f_{j}\right)$ of the standing wave pattern at each frequency $f_{j}$ gives a continuous spectrum with maxima indicating the wave numbers $k_{i, j}^{\max }$ of the modes present in the material. The phase velocities are then simply obtained by $v=\omega / k_{i, j}^{\max }$. The phase velocities of the maxima that have been identified are indicated in Table I. The highest velocities in Table I correspond to the peaks with smaller values of wave number, but these modes can be accounted for only when their wavelengths are sufficiently smaller than the size of the sample. The differences in peak amplitudes are due to differences in the mode amplitudes and to the fact that the detection takes place in the normal direction. The modes with the highest amplitudes are those with main displacements along the $y$ axis. The source configuration plays a role in the excitation efficiency of the modes. The source/material impedance matching is also of great importance in the uniformity of the source amplitude with frequency. The key advantages of this method are that more energy can be delivered at a given frequency and that the signals are continuous, not localized in time. Since the excitation is a continuous sine, the rigid backing of the sample is used in order to create a spatial profile of the sample surface near the reflector that does not depend on time.

However, as can be seen in Fig. 4(c), the standing wave pattern is most clearly observed near the rigid end of the sample and for higher frequencies. This can be explained by the reduced influence of the source in this area and at these
TABLE II. Material parameters for the melamine foam.

\begin{tabular}{cccccc}
\hline \hline & Flow & Viscous & Thermal & Frame & \\
& resistivity & dimension & dimension & density & \\
Tortuosity & Ns $/ \mathrm{m}^{4}$ & $\mu \mathrm{m}$ & $\mu \mathrm{m}$ & $\mathrm{kg} / \mathrm{m}^{3}$ & $\begin{array}{c}\text { Porosity } \\
\alpha_{\infty}\end{array}$ \\
$\sigma$ & $\Lambda$ & $\Lambda^{\prime}$ & $\rho$ & $\phi$ \\
\hline 1.01 & 12000 & 100 & 150 & 13.96 & 0.98 \\
\hline \hline
\end{tabular}

frequencies. The waves are mainly propagative near the source and standing near the rigid end. In this article, the attention is focused on the detection of the spatial periodicities that appear in the standing wave field created by the reflection. At low frequencies, the waves traveling toward and reflected from the rigid end have a fairly low attenuation, and the influence of the source can extend over long distances. At higher frequencies, the attenuation is greater and higher excitation amplitudes are necessary. In practice, overheating of the source and nonlinear effects in the material near the source are limitations to the maximum amplitude applicable.

To isolate the "standing" part of the wave pattern near the rigid end, and to reduce the source effect, a spatial window can be used when calculating the spatial Fourier transform. This window is designed to reduce the amplitudes of the displacement profile near the source. The source effect manifests itself by the presence of dominant peaks of amplitude at low wave numbers in the spatial spectrum. The length of the spatial window should also vary when the frequency varies, as the spatial extent of the source effect changes with frequency. Because it is quite difficult yet to find a reliable guideline for applying a window at varying frequencies, the data were processed twice, with and without window; only the most certain points were retained. Thus, the dominant source effect can be filtered out to obtain the modes propagating with a small wavelength. As can be seen in Fig. 4(d), small ripples are present in the spectrum. These are a consequence of the finite sample dimensions and of the discontinuities introduced in the periodization in the numerical implementation of the Fourier transform. A second function of spatially windowing the standing wave pattern is to reduce the amplitude of these ripples.

\section{APPLICATION TO A HIGHLY POROUS MATERIAL AND FITTING OF THE COMPLEX SHEAR MODULUS AND POISSON RATIO}

\section{A. Theoretical dispersion curves}

A highly porous melamine foam with a thickness of 10 $\mathrm{cm}$ (the material parameters are given in Table II) was studied theoretically and experimentally using the technique described in the previous section. Calculated dispersion curves for this material are presented in Fig. 5. The real parts of the phase velocities of the modes are given in Fig. 5(a) and the imaginary parts in Fig. 5(b). A shear modulus $\mu$ of 110000 $\mathrm{Pa}$, obtained from the high-frequency limit of the experimental results, and a Poisson ratio $\nu$ of 0.25 were used in the simulation. Imaginary parts of $10 \%$ of their respective real parts were added to these coefficients to simulate structural damping. The simulation includes both the Biot attenuation 


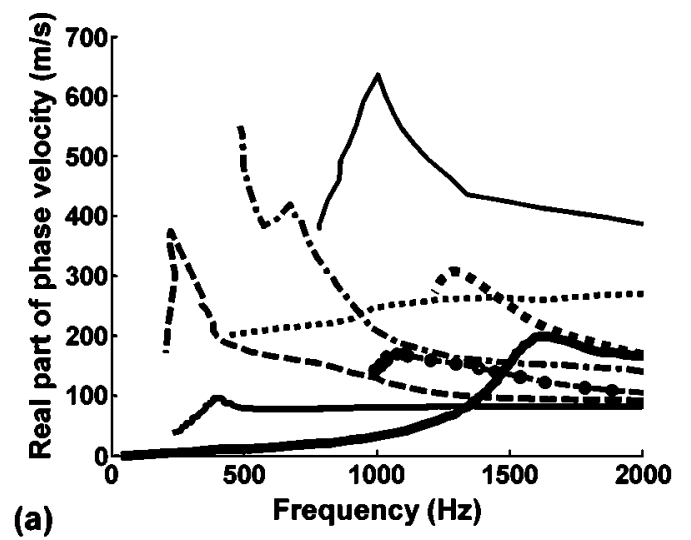

(a)

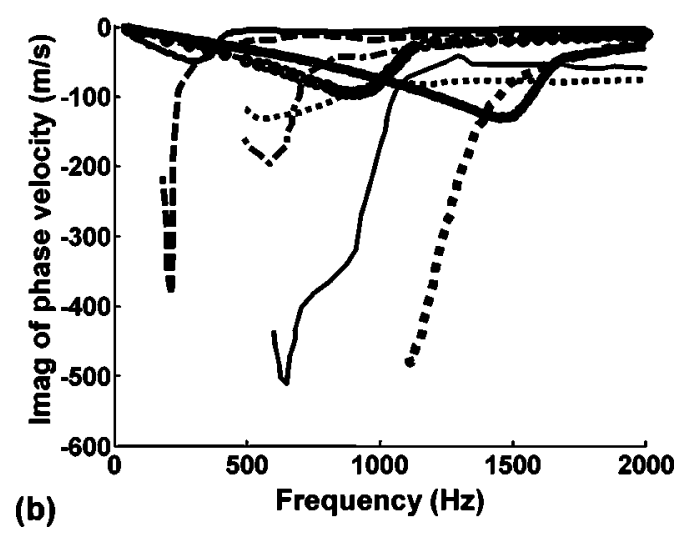

FIG. 5. Dispersion curves calculated by solving Eq. (30) numerically for a melamine foam of 0.1-m thickness. (a) Real part and (b) imaginary part of the phase velocities. The anomalous behavior of the dashed-curve mode around $250 \mathrm{~Hz}$ is due to numerical error.

mechanism in a porous medium and the structural damping associated with complex Young and shear moduli. The damped modes were calculated by searching the zeros of the determinant (29) in the complex plane (no attenuation mechanism was considered in Sec. II A and undamped modes were obtained from a root search on the real axis). A simple numerical algorithm was developed that seems to give good results. The idea is to locate the maxima of the $|\operatorname{Det}(\omega, k, H, \ldots)|^{-1}$ surface in the complex $k$ plane, which is equivalent to solving (30) but with the advantage that this function is real-valued and easier to handle. Numerically, this function can be represented by a large matrix, any value of which has a row and a column index that correspond to the discretized real and imaginary parts of $k$. The root search was done in a MATLAB routine where a smaller square matrix was used to "scan" the large matrix of data to find the local maxima. The size of this "scanning" matrix can be chosen so that there is only one maximum in it. The condition that the maximum found in the scanning matrix must not be on its edges must be fulfilled because it does not necessarily correspond to a local maximum of the large matrix. Several simple methods can be used to implement this condition numerically. This zero search algorithm is simple and fast enough for our study. Figure 6 shows an example of contour plot of the function $|\operatorname{Det}(\omega, k, H, \ldots)|^{-1}$ at $2000 \mathrm{~Hz}$ for a melamine foam as an example of root search in the complex $k$ plane. The sign convention used for $k$ in the numerical algorithm was $k=\operatorname{Re}(k)-i \operatorname{Im}(k)$, and the lower-right quad-

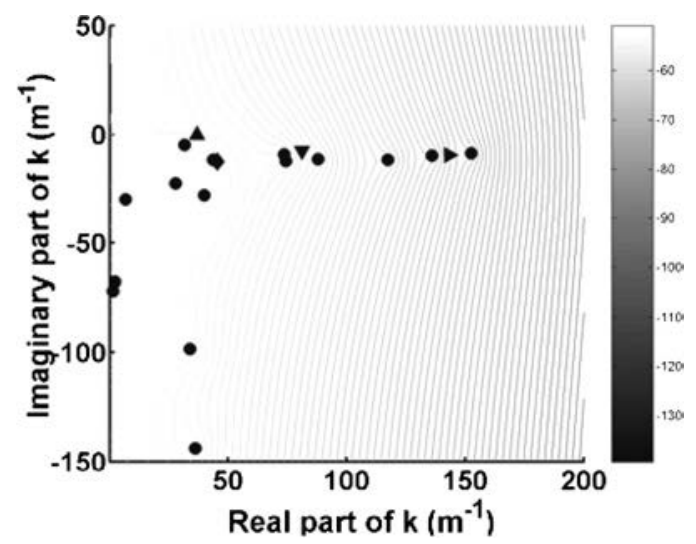

FIG. 6. Root search of Eq. (30) in the complex $k$ plane at $2000 \mathrm{~Hz}$. The spots correspond to the roots found numerically. The downward triangle, the diamond, the right and the upward triangle correspond, respectively, to the first, to the second longitudinal Biot wave number, to the transverse Biot wave numbers, and to the fluid wave number.

rant of the $k$ space was used. The dots correspond to the maxima found, i.e., the roots of Eq. (30). The downward triangle, the diamond, the right- and the upward triangles are trivial solutions of Eq. (30). They correspond, respectively, to Biot's first and second compressional wave numbers, to Biot's shear wave number, and to the fluid wave number (these modes are not shown in Fig. 5). In addition, each mode was calculated separately by using a branch-following algorithm in which a root is being traced from high to low frequencies.

\section{B. Interpretation of the theoretical results}

When comparing the results with those obtained from the results of Sec. II A, in which the porous solid is considered as an effective nonporous solid, it is found that more modes exist in the porous case. This result can be associated with the existence of the fluid and solid phases. The interpretation can be made easier by considering, in a first approach, the purely theoretical case of a porous layer with a rigorously zero coupling between the two Biot waves. In such a case, one may consider two decoupled sets of guided waves in the porous layer: the structure-borne guided waves and the fluidborne guided waves. If Biot's structure-borne bulk waves (compressional and shear) were nondispersive at all, the structure-borne set of guided modes in the layer would correspond exactly to the modes obtained from the effective solid model of Sec. II A. The new result is the possible existence of a second set of guided modes associated with $\mathrm{Bi}$ ot's fluid-borne wave. This result is a direct consequence of the addition of the term containing the scalar potential $\varphi_{2}$ associated with Biot's wave of the second kind in Eq. (9). Including this potential results in increasing the number of solutions for the dispersion equation and therefore the number of modes in the porous system. The porous material studied in this article is such that the coupling between the two compressional waves is weak, and so it is thought that the interpretation given above should hold for this material. However, the coupling, even though very small, always exists in real experimental conditions and it is thought that the main effect of the coupling will be the possibility for the 


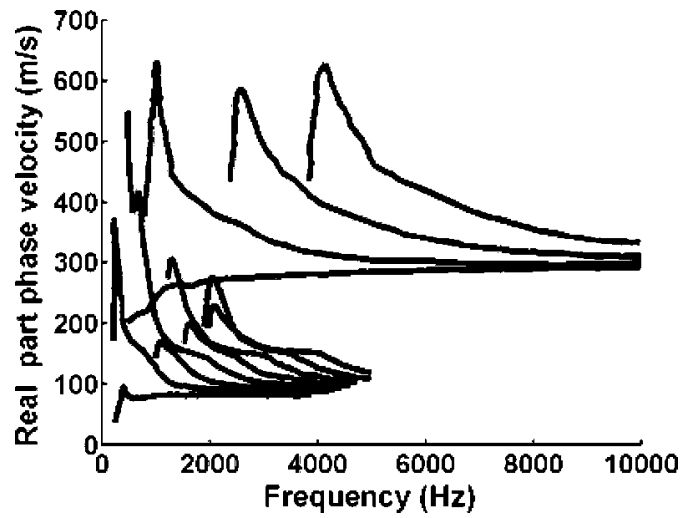

FIG. 7. Real part of the dispersion curves calculated as a function of frequency for a melamine foam of $0.1 \mathrm{~m}$ thickness up to $10 \mathrm{kHz}$.

structure- and fluid-borne guided waves to exchange energy. It is difficult to envisage the detection of any of the fluidborne modes at the moment with the current experimental setup designed to measure the displacement of the solid structure only.

Another consequence of the porous nature of the layer is the possible loss of energy by viscous and thermal interactions in the pores resulting in a higher attenuation for the guided modes in the porous material than for an equivalent elastic solid in which only structural damping is accounted for. On average and for all the modes, about $15 \%$ extra damping should be attributed to Biot's attenuation in the dispersion curves of Fig. 5. While this result holds only for the porous material studied in this article, Biot's attenuation should in general affect both the damping and the dispersion of the modes. In addition to the existence of the fluid-borne dispersion curves, the existence of a second compressional wave in a porous medium has a very interesting consequence. As frequency increases, it can be seen in Fig. 5(a) that the thin dotted line and the thin solid line tend to the velocity of the Biot wave of the second kind. In the material studied, the Biot wave of the second kind is faster than the Biot wave of the first kind at high frequencies, and is slower than the free velocity in the surrounding fluid. The curves are plotted in a wider frequency range in Fig. 7, clearly showing the two sets of dispersion curves. The shear velocity acts as asymptotic values toward which the structure-borne modes tend. While this behavior can be expected in a nonporous solid, the existence of a second velocity limit in porous media corresponding to the velocity of the Biot wave of the second kind is new. This behavior can be related to the fact that the fluid-borne modes propagate in an equivalent "fluid plate." In such a plate, the shear velocity is zero and the modes tend to the bulk compressional velocity in the highfrequency limit. ${ }^{30}$ The existence of a compressional and a shear limit velocity can also be observed in materials for which the coupling between the compressional and shear waves is small, such as in highly attenuative plastic plates. ${ }^{31}$ The attenuation mechanisms and the presence of a loading fluid are responsible for the existence of the maxima observed in the dispersion curves of Figs. 5 and 7.

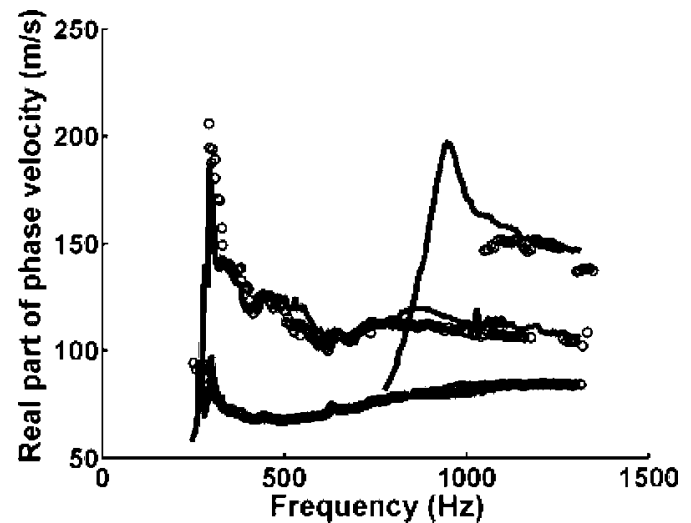

FIG. 8. Measured phase velocities (circles) and fitted modes (solid lines) for a melamine foam.

\section{Fitting of the dispersion curves}

The new experimental method was used to determine the phase velocities in a layer of melamine foam. The sample was set up as shown in Fig. 3 and a continuous sine excitation with frequencies varying from 150 to $1500 \mathrm{~Hz}$ was applied. The measurements were performed at a temperature of $21^{\circ} \mathrm{C}$ and variations remained within $5 \%$ of this value. Two clear maxima could be traced throughout the measured frequency domain. These provided the velocities of the first two modes of the dispersion curves. The results are shown in Fig. 8. Several data points seem to provide evidence for the existence of a third mode. It was found that a variation in frequency of the elastic coefficients and the use of the complete porous model was needed to fit the variations of the first two modes. The solid lines indicate a fit where the shear modulus and its imaginary part were allowed to vary. The parameters for the fit are the real and imaginary parts of the shear modulus and the Poisson ratio. All other physical parameters: porosity, permeability, tortuosity, thermal and viscous characteristic lengths, were measured. Standard methods exist for the first two parameters. The last three parameters were determined using ultrasonic transmission methods (see Ref. 32 and references therein). In the fitting process, the experimental results were assumed to correspond to the first two modes (indicated by the medium thick solid line and the dashed line) of Fig. 5(a). This indirectly provided a guess for the initial value of the shear modulus as the first mode tends asymptotically to the Rayleigh velocity, which depends on the shear modulus. A separate measurement of the Rayleigh velocity at high frequency was performed on a smaller sample of the same material and was consistent with the high-frequency asymptotic limit. An initial value of 0.25 was used for the Poisson coefficient. The imaginary part of the Poisson coefficient was allowed to vary within $20 \%$ of the real part. The values obtained for the shear modulus, the Poisson coefficients, and their imaginary parts are given in Fig. 9. A variation of the shear modulus between 0.05 and $0.12 \mathrm{GPa}$ was obtained in the frequency range investigated. Although the measurement error is difficult to estimate precisely, the discrepancy between the fit and the measured phase velocities gives an indication. It was noticed that the fit is less sensitive to the Poisson ratio than to the 


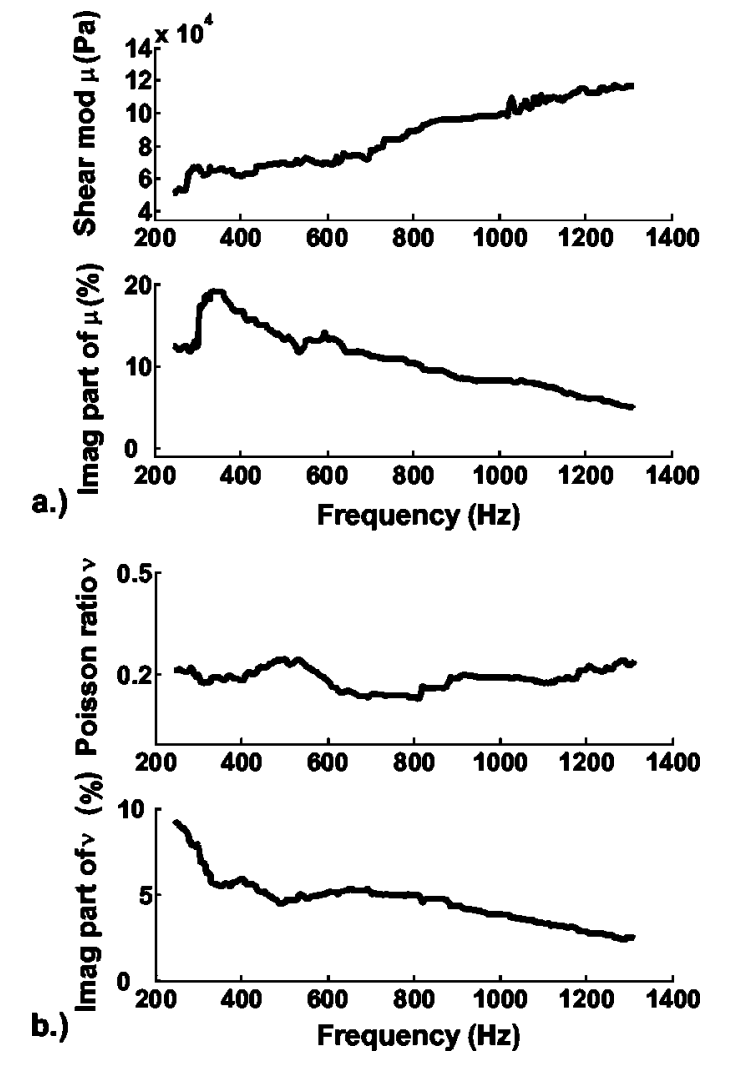

FIG. 9. Fitted real and imaginary parts of the shear modulus and Poisson ratio against frequency. The imaginary parts are plotted in percentage of the real parts.

shear modulus. As a consequence, one may consider the result to be reliable for the shear modulus while only giving an order of magnitude for the variations of the Poisson ratio.

Although precise literature values on the variation of the shear modulus for this type of highly porous foams are scarce, the values measured in this study can be compared to other measurements. ${ }^{9,10}$ In Ref. 10, vibration measurements on this type of foam (melamine) gave a range of variation for the Young's modulus between $1.5 \times 10^{5} \mathrm{~Pa}$ and $2.3 \times 10^{5} \mathrm{~Pa}$ between 200 and $1000 \mathrm{~Hz}$. Considering a real part of Poisson ratio around 0.2 [obtained from the fit in Fig. 9(b)], the corresponding range for the shear modulus is between 6.2 $\times 10^{4} \mathrm{~Pa}$ and $9.6 \times 10^{4} \mathrm{~Pa}$. In the same frequency range, the results we obtained vary between $7 \times 10^{4} \mathrm{~Pa}$ and 10 $\times 10^{4} \mathrm{~Pa}$ [real part of the shear modulus in Fig. 9(a)], showing a good consistency with the results of Ref. 10 and a difference between $4 \%$ and $11 \%$. The error on the Poisson ratio should also be added when carrying out the comparison. This type of material exhibits a non-negligible anisotropy, and the way the material is cut can explain the differences observed. However, the range of values obtained is confirmed by static tests, by the traditional lower frequency measurement methods, and by the Rayleigh wave measurement method ${ }^{11}$ that was also performed on this material in the high-frequency limit.

\section{CONCLUSION}

A new experimental technique for measuring the dynamic complex shear modulus and Poisson ratio of porous materials was proposed and applied to a highly porous melamine foam. This method is based on the generation of standing waves in the layer and accounts for the dispersion of guided acoustic waves in the layer of material lying on a rigid substrate. The dispersion of the modes was also studied theoretically and new, interesting results were found on the consequences of the existence of two compressional waves in a porous material. The coupling between the two Biot compressional waves is fairly low in the material studied, and structure-borne and fluid-borne guided waves were predicted. It was also found that the velocity of the Biot compressional wave of the second kind acts as a limit velocity for the fluid-borne dispersion curves, and that a significant additional damping was induced by the inclusion of Biot's parameters in the model.

The experimental results are consistent with other measurements on this material and with the results obtained from static tests and traditional methods. Although this method allows description of a higher frequency range than the traditional methods, it is still limited by the lack of a reliable line source that can be used to excite high-frequency vibrations with sufficient amplitude. Other materials, experimental configurations, theoretical development, as well as new excitation techniques are currently being investigated. In particular, a porous layer with its two surfaces free (Lamb conditions) is being studied experimentally and theoretically. This work should allow the study of more rigid materials and the exploration of a higher frequency range.

\section{APPENDIX:}

The elements of the determinant in Eq. (29) are given by

$D_{12}=-2 i k p_{1}$,

$D_{14}=-2 i k p_{2}$,

$D_{15}=k^{2}-q^{2}$,

$D_{21}=-2 \mu p_{1}^{2}-\left(k^{2}+p_{1}^{2}\right)\left(\lambda_{c}+\alpha M \phi\left(\mu_{1}-1\right)\right)$,

$D_{23}=-2 \mu p_{2}^{2}-\left(k^{2}+p_{2}^{2}\right)\left(\lambda_{c}+\alpha M \phi\left(\mu_{2}-1\right)\right)$,

$D_{26}=2 i k q \mu$,

$D_{27}=-K_{f}\left(-k^{2}+\gamma^{2}\right)$,

$D_{31}=\left(k^{2}+p_{1}^{2}\right) M\left(\alpha+\phi\left(\mu_{1}-1\right)\right)$,

$D_{33}=\left(k^{2}+p_{2}^{2}\right) M\left(\alpha+\phi\left(\mu_{2}-1\right)\right)$,

$D_{37}=K_{f}\left(-k^{2}+\gamma^{2}\right)$,

$D_{42}=p_{1}\left(1+\phi\left(\mu_{1}-1\right)\right)$,

$D_{44}=p_{2}\left(1+\phi\left(\mu_{2}-1\right)\right)$,

$D_{45}=i k\left(1+\phi\left(\mu_{3}-1\right)\right)$,

$D_{47}=-\gamma$,

$D_{51}=-p_{1} \sin p_{1} H$,

$D_{52}=p_{1} \cos p_{1} H$,

$D_{53}=-p_{2} \sin p_{2} H$,

$D_{54}=p_{2} \cos p_{2} H$,

$D_{55}=i k \cos q H$,

$D_{56}=i k \sin q H$,

$D_{61}=-i k \cos p_{1} H$,

$D_{62}=-i k \sin p_{1} H$,

$D_{63}=-i k \cos p_{2} H$,

$D_{64}=-i k \sin p_{2} H$,

$D_{65}=-q \sin q H$,

$D_{66}=q \cos q H$,

$D_{71}=-\phi\left(\mu_{1}-1\right) p_{1} \sin p_{1} H$, 


$$
\begin{aligned}
& D_{72}=\phi\left(\mu_{1}-1\right) p_{1} \cos p_{1} H, \\
& D_{73}=-\phi\left(\mu_{2}-1\right) p_{2} \sin p_{2} H, \\
& D_{74}=\phi\left(\mu_{2}-1\right) p_{2} \cos p_{2} H, \\
& D_{75}=i \phi\left(\mu_{3}-1\right) k \cos q H, \\
& D_{76}=i \phi\left(\mu_{3}-1\right) k \sin q H .
\end{aligned}
$$

${ }^{1}$ M. A. Biot, "Theory of propagation of elastic waves in a fluid-saturated porous solid," J. Acoust. Soc. Am. 28, 168-191 (1956).

${ }^{2}$ J. F. Allard, Propagation of Sound in Porous Media: Modeling Sound Absorbing Materials (Chapman \& Hall, London, 1993).

${ }^{3}$ A. Bardot, B. Brouard, and J. F. Allard, "Frame decoupling at low frequency in thin porous layers saturated by air," J. Appl. Phys. 79, 82238229 (1996).

${ }^{4}$ J. F. Allard, C. Depollier, Ph. Guignouard, and P. Rebillard, "Effect of resonance on the surface impedance of glass wool of high density and thickness," J. Acoust. Soc. Am. 89, 999-1001 (1991).

${ }^{5}$ T. Pritz, "Transfer function method for investigating the complex modulus of acoustic materials: Rod-like specimen," J. Sound Vib. 81, 359-376 (1982).

${ }^{6}$ T. Pritz, "Frequency dependence of frame dynamic characteristics of mineral and glass wool materials," J. Sound Vib. 106, 161-169 (1986).

${ }^{7}$ T. Pritz, "Dynamic Young's modulus, and loss factor of plastic foams for impact sound isolation," J. Sound Vib. 178, 315-322 (1994).

${ }^{8}$ T. Pritz, "Measurement methods of complex Poisson's ratio of viscoelastic materials," Appl. Acoust. 60, 279-292 (2000).

${ }^{9}$ A. Sfaoui, "On the viscoelasticity of the polyurethane foam," J. Acoust. Soc. Am. 97, 1046-1052 (1995).

${ }^{10}$ L. Jaouen, "Contribution à la caractérisation mécanique de matériaux poro-viscoélastiques en vibro-acoustique," (Contribution to the mechanical characterization of poroviscoelastic materials in vibroacoustics), Ph.D. thesis, Université de Sherbrooke, Canada, 2003.

${ }^{11}$ J. F. Allard, G. Jansens, G. Vermeir, and W. Lauriks, "Frame-borne surface waves in air-saturated porous media," J. Acoust. Soc. Am. 111, 690696 (2002).

${ }^{12}$ J. F. Allard, M. Henry, L. Boeckx, P. Leclaire, and W. Lauriks, "Acoustical measurement of the shear modulus for thin porous layers," J. Acoust. Soc. Am. (submitted).

${ }^{13} \mathrm{H}$. Deresiewicz, "The effect of boundaries on wave propagation in a liquid-filled porous solid. IV. Surface waves in a half space," Bull. Seismol. Soc. Am. 52, 627-638 (1962).

${ }^{14}$ S. Feng and D. L. Johnson, "High-Frequency acoustic properties of a fluid/porous solid interface. I. New surface mode," J. Acoust. Soc. Am. 74, 906-914 (1983).

${ }^{15} \mathrm{~K}$. Attenborough, "Review of ground effects on outdoor sound propaga- tion from continuous broadband sources," Appl. Acoust. 24, 289-319 (1988).

${ }^{16} \mathrm{~K}$. Attenborough, "Solid particle motion induced by a point source above a poroelastic half-space," J. Acoust. Soc. Am. 86, 1085-1092 (1989).

${ }^{17} \mathrm{~K}$. Attenborough and Y. Chen, "Surface waves at an interface between air and an air-filled poroelastic ground," J. Acoust. Soc. Am. 87, 1010-1016 (1990).

${ }^{18}$ W. Lauriks, L. Kelders, and J. F. Allard, "Surface waves and leaky waves above a porous layer," Wave Motion 28, 57-67 (1998).

${ }^{19}$ L. Kelders, W. Lauriks, and J. F. Allard, "Surface waves above thin porous layers saturated by air at ultrasonic frequencies," J. Acoust. Soc. Am. 104, 882-889 (1998).

${ }^{20}$ J. F. Allard, M. Henry, C. Glorieux, S. Petillion, and W. Lauriks, "Laserinduced surface modes at an air-porous medium interface," J. Appl. Phys. 93, 1298-1304 (2003).

${ }^{21}$ L. Boeckx, P. Leclaire, C. Glorieux, W. Lauriks, and J. F. Allard, "Measuring the dynamic shear modulus of poroelastic foams in the audible frequency range," in Proceedings International Congress in Acoustics, Kyoto, 4-9 April 2004.

${ }^{22}$ D. E. Chimenti, "Guided waves in plates and their use in materials characterization,” Appl. Mech. Rev. 50, 247-284 (1997).

${ }^{23}$ D. Royer, and E. Dieulesaint, Elastic Waves in Solids: Free and Guided Propagation (Springer, Berlin, 1999), Vol. 1.

${ }^{24}$ I. A. Viktorov, Rayleigh and Lamb Waves (Plenum, New York, 1967).

${ }^{25}$ W. M. Ewing, W. S. Jardetsky, and F. Press, Elastic Waves in Layered Media (McGraw-Hill, New York, 1957).

${ }^{26} \mathrm{~V}$. Gusev and P. Hess, "Theory of photothermal depth profiling via Rayleigh-type surface-acoustic-wave detection," Appl. Phys. A: Mater. Sci. Process. 61, 299-310 (1995).

${ }^{27} \mathrm{~K}$. Van de Rostyne, "Laser ultrasonic study of wave propagation at fluidsolid interfaces," Ph.D. thesis, Katholieke Universiteit Leuven, Belgium, 2001

${ }^{28}$ M. A. Biot, "Mechanics of deformation and acoustic propagation in porous media," J. Appl. Phys. 33, 1482-1498 (1962).

${ }^{29}$ M. A. Biot and D. G. Willis, "The elastic coefficients of the theory of consolidation," J. Appl. Mech. 24, 594-601 (1957).

${ }^{30}$ H. Überall, B. Hosten, M. Deschamps, and A. Gérard, "Repulsion of phase-velocity dispersion curves and the nature of plate vibrations," J. Acoust. Soc. Am. 96, 908-917 (1994).

${ }^{31}$ C. W. Chan and P. Cawley, "Lamb waves in highly attenuative plastic plates," J. Acoust. Soc. Am. 104, 874-881 (1998).

${ }^{32}$ P. Leclaire, L. Kelders, W. Lauriks, M. Melon, N. Brown, and B. Castagnède, "Determination of the viscous and thermal characteristic lengths of plastic foams by ultrasonic measurements in helium and air," J. Appl. Phys. 80, 2009-2012 (1996). 